\title{
Pain Comorbidities: Understanding and Treating the Complex Patient
}

\author{
Maria Adele Giamberardino, Troels Staehelin Jensen. IASP Press, Seattle, WA, \\ Year of Publication: 2012; Number of pages: 507; ISBN 978-0-931092-92-3
}

\begin{abstract}
Akilan Velayudhan, MBBS
Received: 11 December 2012/ Accepted: 18 December 2012/Published online: 4 January 2013

(C) Canadian Anesthesiologists' Society 2013
\end{abstract}

This publication by IASP Press provides readers with a comprehensive update on chronic pain and its multiple interactions with medical comorbidities. The editors are leading experts in complex pain states, such as neuropathic pain, fibromyalgia, and headache, and the contributing authors have a range of expertise that spans from basic science research to clinical aspects of pain, including related physiotherapy and psychology considerations.

The 41 contributing authors are based in Europe, North America, and Australia, and all are international experts on the topics presented in this publication. The authors review various clinical conditions to provide an understanding of the interactions of these conditions with multiple concurrent diseases and to offer guidance for their management.

The book is divided into three parts. The focus of Part I is on the general aspects of pain, including epidemiology, and includes an introduction to various animal and human models of pain and non-pain comorbidities. Part II is a review of the clinical aspects of concurrent pain and nonpain conditions, and Part III involves management aspects of pain and non-pain comorbidities.

The introductory section of the book provides background on some historical aspects of pain and includes a discussion on the important relationship between pain and emotions. This section also includes an overview of epidemiological approaches to measure the relationship between chronic pain and non-pain comorbidities. The discussion then extends to experimental animal models of pain and non-pain comorbidities and the possible clinical implications of translating research findings to clinical practice. The following chapter on experimental human

A. Velayudhan, MBBS $(\square)$

University of Western Ontario, London, ON, Canada

e-mail: akilanv@doctors.org.uk models addresses the fundamentals of human quantitative pain assessment and their application in a variety of nonpainful disorders. The focus then turns to the neurobiological approach to comorbid pain conditions with evidence from animal models, genetic factors, hormonal factors, immunological factors, and psychosocial factors. Some of these topics may appeal to researchers as well as to clinicians interested in these areas.

Part II of the book is rather intriguing as it involves clinical aspects of pain and non-pain comorbidities such as hypertension, diabetes, obesity, and metabolic and inflammatory disease. Two well-discussed issues in this section are the alteration of blood pressure-related hypoalgesia in chronic pain patients and the question whether or not chronic pain is a risk factor in the development of hypertension. The chapter on diabetes and associated neuropathic pain is a contemporary and comprehensive summary of the subject matter. Discussions in subsequent chapters involve such topics as the correlation between headaches and cardiovascular disorders, myofascial pain in headache patients, obesity and pain, and visceral pain and headache in fibromyalgia.

The chapter on pain and affective disorders, which is discussed on the basis of the "chicken and egg" conundrum, is very thought provoking and interesting, as the author discusses the relationship between pain and affective disturbances such as anxiety, depression, and anger. The following chapter on viscerovisceral hyperalgesia, which is a relatively neglected phenomenon, is well explored and presented. There is also a chapter on musculoskeletal pain where the author focuses on various forms of primary painful joint disease and muscle pain comorbidities such as myofascial pain syndromes.

In Part III, the third and final section of this book, the principles of pain management in patients with pain 
comorbidities are discussed using multidisciplinary, pharmacological, physical training and rehabilitation, and psychological approaches. The focus of the chapter on pharmacotherapy is on the classes of drugs used in pain treatment and their interactions and side effects and on the importance of carefully assessing comorbidities for pharmacological therapy. Antidepressants are one class of drugs described in detail, and the use of these drugs in pain and comorbid disorders is highlighted. The authors of the chapter on physical training discuss a strategy that needs to be adopted in patients with pain and coexisting comorbidities, particularly those with cardiac and other medical conditions.
The text has an appropriate number of supporting figures, diagrams, and tables, and it includes up-to-date reference lists that afford readers the opportunity to explore individual topics in further detail. In summary, this is an excellent textbook that provides an in-depth review of complex pain states with a focus on patients with coexisting diseases. The information is novel and up-to-date and provides clinicians with a holistic approach to the management of pain patients with extensive comorbidities.

Conflicts of interest None declared. 